International Journal of Computer Networks \& Communications (IJCNC) Vol.8, No.3, May 2016

\title{
Performance ANalysis of THE Link-Adaptive COOPERATIVE AMPLIFY-AND-FORWARD RELAY NETWORKS WITH OPPORTUNISTIC RELAYING STRATEGY
}

\author{
Bhuvan Modi, O. Olabiyi and A.Annamalai \\ Center of Excellence for Communication Systems Technology Research \\ Department of Electrical and Computer Engineering, \\ Prairie View A \& M University, TX 77446 United States of America
}

\begin{abstract}
This paper analyzes the performance of cooperative amplify-and-forward (CAF) relay networks that employ adaptive M-ary quadrature amplitude modulation (M-QAM)/M-ary phase shift keying (M-PSK) digital modulation techniques in Nakagami-m fading channel. In particular, we present and compared the analysis of CAF relay networks with different cooperative diversity and opportunistic routing strategies such as regular Maximal Ratio Combining (MRC), Selection Diversity Combining (SDC), Opportunistic Relay Selection with Maximal Ratio Combining (ORS-MRC) and Opportunistic Relay Selection with Selection Diversity Combining (ORS-SDC). We advocate a simple yet unified numerical approach based on the marginal moment generating function $(M G F)$ of the total received SNR to compute the average symbol error rate (ASER), mean achievable spectral efficiency, and outage probability performance metrics.
\end{abstract}

\section{KEYWORDS}

Cooperative communications, adaptive M-QAM/MPSK modulation, Opportunistic relay selection

\section{INTRODUCTION}

The current and the future network design is highly challenged in every front due to increasing connectivity and data rate requirements. The global internet traffic has experienced exponential growth in the past 10 years and this plummeting growth is expected to continue in the future. Cisco has predicted that annual global internet traffic is expected to reach zettabyte threshold by 2015 from current 15 billion network connections (including machine-to-machine connections) [1]. This means, an average of more than two devices are expected to be in use per person on earth. This surge in connectivity is attributed to the proliferation of the communication devices such as tablets, mobile phones, connected appliances, and other smart machines. Since most of these devices are mobile in nature, the increased connectivity requirement will be placing a huge demand on already limited wireless network access resources. Also, as most of the predicted internet traffic is expected to be dominated by video contents, there is a need to find more cost effective ways of delivering these high data rate services to the end users within the limited wireless channel bandwidth. Therefore, the development of very high-speed wireless access system is imperative. Most of the ongoing communication research and industrial standard efforts are dedicated to solving this problem. In fact the evolution of mobile networks from $2 / 2.5 \mathrm{G}$ (GSM, GPRS, EDGE, IS95/IS98) to 3G (WCDMA/HSPA/CDMA2000) and to 4G (LTE/ $\mathrm{HSPA}+/$ WIMAX) has been in response to address this issue [2]. While the current $4 \mathrm{G}$ access 
network holds the promise of delivering up to 1GBps data rate to end users (mostly available at the macrocell base station), the proliferation of mobile devices has lead to very small-size "hot spot" and therefore require extensive dimensioning of network resources in terms of coverage. These hot spots could be homes, trains, airports and possibly buses with high data rate wireless access requirements. The range extension (with high speed connectivity) has been a major point of interest in LTE advance standard, and femtocells (very small base stations installable by the end users) have been identified as the most promising potential solution. Since femtocells will act as relays between the end user and the macrocells (regular base station) with multiple femtocells expected to cooperate, the $4 \mathrm{G}$ network can greatly benefit from the ongoing research on the new communication paradigm of cooperative relay system. The deployment of a large number of femtocells can then be used to improve coverage, capacity (area and system spectral efficiency), and energy efficiency of the wireless network access system.

The cooperative relay communication system takes advantage of the broadcast nature of the wireless channel to improve the communication between the source node and a destination node with the aid of one or more relay nodes. The system harnesses the new form of spatial diversity and combat multipath fading, thereby improving the spectral efficiency, and reducing the transmission error, system outages, and transmission power. The reduced transmission power (of wireless device, femtocell and macrocell) effectively lowers the inter and intra macrocell interference, thereby improving both the system and the area spectral efficiency. In another development, the "cooperative diversity system" concept has gained research impetus owing to its inherent ability to overcome the practical implementation issue of packing a large number of antenna elements (to exploit the benefits of multiple-input-multiple-output (MIMO) space-time processing techniques) in small form-factor devices. In general, there are three cooperative relaying protocols: amplify-and-forward, decode-and-forward, and compress-and-forward [3-7].

The other variations include incremental, opportunistic, blind and semi-blind relays. In this article, we advocate the implementation of the amplify-and-forward protocol on femtocell. The advantages of this include its simplicity, lower implementation cost (i.e., relay nodes (femtocells) do not have to decode and then re-encode the information received prior transmission) and possibly the better privacy. The main drawback of the regular cooperative amplify-and-forward (CAF) diversity system which employs the maximum ratio combining (MRC) or selection diversity combining (SDC) at the destination's receiver is that each relay has to transmit on the orthogonal channels (TDMA/CDMA). Therefore, the spectral efficiency is scaled by $1 /(N+1)$, where $N$ is the number of relays, which reduces the capacity with increasing number of relays. In order to improve this, Bletsas et al. and Zhao et al. [8-11], proposed relay selection method otherwise known as the opportunistic relaying system (ORS). Here, the best relay is selected prior to relay-to-destination transmission to limit the number of orthogonal transmissions to two. The destination would then employ either MRC (subsequently referred to as ORS-MRC) or SDC (subsequently referred to as ORS-SDC) diversity scheme on the two final diversity paths. In addition to reducing the number of independent transmissions, the ORS protocols have been shown to achieve full diversity just like regular relay system [27].

Adaptive transmission is yet another powerful wireless communication strategy for improving the spectral utilization efficiency, wherein the signal constellation size, power level and/or the coding rate are "matched" to the prevailing channel conditions based on the acquired channel-sideinformation (CSI) on the feedback channel. Several articles have investigated the combination of the link adaptation and the regular cooperative diversity system, both from theoretical limit (ergodic capacity) and practical implementation (using digital modulation schemes) perspectives. 
For instance, the ergodic capacity of cooperative amplify-and-forward (CAF) relay networks with the limited CSI were derived in [12-19] for different source-adaptive transmission policies in a myriad of stochastic fading environments. The performance of practical adaptive digital modulation scheme with regular relay system was considered in [20 -23]. The performance of $\mathrm{CAF}$ relay with constant power M-QAM adaptive rate transmission, when average bit error rate (ABER) in Rayleigh fading is constrained to be below a specified target bit error rate (BER) is examined in [21] and [22] for fixed and optimum mode switching thresholds, respectively. In [23], the performance of discrete-rate adaptive M-QAM for a single incremental relay in Nakagami-m environment was examined, while [22] investigates the performance of a cooperative decode-and-forward relay network with five-modes adaptive M-QAM transmission in an i.i.d Nakagami-m wireless fading environment. All these articles and related references indicate the advantage of the adaptive cooperative diversity system over the non-adaptive and/or the non-cooperative system especially at low and medium signal-to-noise ratio (SNR). However, the half-duplex nature of regular relay system makes the performance worse at high SNRs.

With the introduction of ORS in [8-11], several articles have been published on its performance, but mostly focusing on non-adaptive system (i.e. fixed modulation). Average symbol or bit error rate (ASER/ABER) performance for ORS-MRC scheme over Rayleigh, independent and identically distributed (i.i.d) Nakagami-m, and independent and non-identically distributed (i.n.d) Nakagami-m fading channels was investigated in [26-27], [28], and [29] respectively, while [30] investigated ASER of both ORS-MRC and ORS-SDC scheme over i.i.d Nakagami-m fading channel. Outage capacity for ORS-MRC and ORS-SDC has been considered in [31]. It is important to note that until now; only a few articles have considered the link adaptive ORS system. For instance, ergodic capacity with the source adaptation techniques have been considered in [32-35] for the ORS-MRC scheme over Rayleigh fading channel, while the variable rate constant power adaptive M-QAM modulation with ORS-MRC and ORS-SDC schemes over Rayleigh fading have been considered in [35-36] and [37] respectively. Also, [38] analyzed the performance of link adaptive incremental opportunistic relaying over i.n.d Rayleigh fading channels. However [39] studied the performance analysis of cooperative communication with only ORS-SDC scheme. Careful study of the adaptive ORS schemes [32-39] indicates that, the analyses of both the ergodic capacity and the practically achievable spectral efficiency have been obtained using the probability density function method, which can be very tedious and may not always yield compact solutions.

In contrast, in this article we develop a new analytical framework based on the marginal MGF method for evaluating the ASER, mean spectral utilization efficiency and outage probability performance metrics (i.e., since the MGF of total received SNR may be easier to compute or readily available for CAF relay networks).The developed analytical framework is thenused to analyze and compare the performance of regular MRC, SDC, opportunistic MRC and SDC CAF relay schemes that employ constant power adaptive discrete rate M-QAM/M-PSK transmission.

The proposed analytical framework is general, and can be applied to any arbitrary fade distribution as long as the MGF of the end-to-end SNR is available unlike channel specific derivations in [32-38]. For completeness purpose, the ergodic capacity with optimal rate adaptation was also presented. Numerical analysis indicates that ORS-MRC leads the pack considering the ergodic capacity and achievable spectral efficiency, while the regular MRC is the best in terms of the outage probability and ASER. In the overall, the ORS-MRC is the best due to the additional power saving as it consumes only $2 /(\mathrm{N}+1)$, of the total power consumed by regular MRC. Therefore, not only does it perform well as a communication paradigm, it also supports green technology due to its low transmit power requirement. Careful literature search indicates that, this is perhaps the first time such comprehensive analysis of regular and opportunistic CAF relay network with the link adaptation is being reported. 
The remainder of this article is organized as follows. In Section 2, the system model is discussed. Section 3 derives the performance metrics for the CAF relay networks with adaptive M-QAM/MPSK modulation. Selected numerical results are presented in Section 4. Our conclusions are given in Section 5.

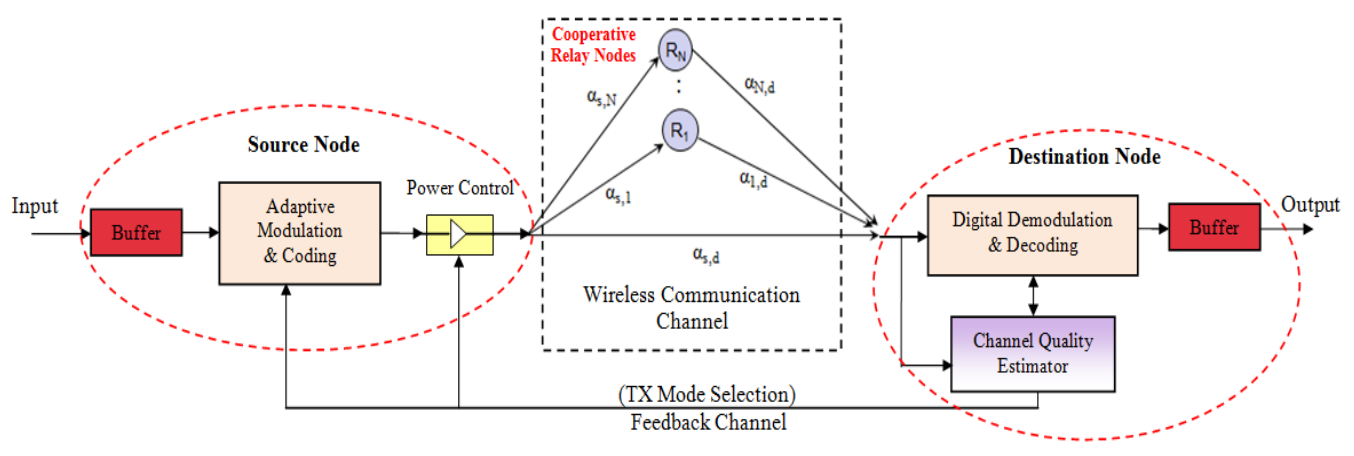

Figure 1. Link-adaptive cooperative relay networks

\section{SYSTEM MODEL}

\subsection{Total MGF Of CAF Relay Networks}

In this section, we will present the moment generating function (MGF) of the relayed path of different cooperative diversity and opportunistic routing protocols that will be utilized in evaluating the end-to-end ASER, mean spectral efficiency and the outage probability performance metrics of the proposed network over a myriad of fading channels with the adaptive M-QAM/M-PSK modulation schemes.

\subsubsection{Regular Cooperation: CAF Relaying With Maximum Ratio Combining (MRC) AT THE DESTINATION}

In this protocol, as shown in Fig 1, source node $\mathrm{S}$ which communicates with a destination node $\mathrm{D}$ via a direct-link and through $\mathrm{N}$ amplify-and-forward relays, $\mathrm{R}_{\mathrm{i},} i \in\{1,2, \ldots, N\}$, in two transmission phases. During the initial Phase I, $S$ transmits signal $x$ to $D$ as well as to the relays $R_{i}$, where the channel fading coefficients between $S$ and $D, S$ and the i-th relay node $R_{i}$, and $R_{i}$ and $D$ are denoted by $\alpha_{s, d}, \alpha_{s, i}$ and $\alpha_{i, d}$, respectively. During the second phase of cooperation, each of the $\mathrm{N}$ relay nodes transmits the received signal after amplification via orthogonal transmissions (e.g., TDMA in a round-robin fashion and/or FDMA). Hence, the channel usage per source transmission, $U(N)=N+1$.

Now, consider that the maximum ratio combiner (MRC) is employed at a $D$ to coherently combine all the signals received during Phase I and Phase II, the total received SNR at output of the MRC detector can be shown to be (e.g., $[19,20,25])$

$\gamma_{T}^{M R C}=\gamma_{s, d}+\sum_{i=1}^{N} \frac{\gamma_{s, i} \gamma_{i, d}}{\gamma_{s, i}+\gamma_{i, d}+1}=\gamma_{s, d}+\sum_{i=1}^{N} \gamma_{i} \leq \gamma_{s, d}+\sum_{i=1}^{N} \gamma_{i}^{(H M)}$ 
International Journal of Computer Networks \& Communications (IJCNC) Vol.8, No.3, May 2016

where $\gamma_{i}^{(H M)}=\gamma_{s, i} \gamma_{i, d} / \gamma_{s, i}+\gamma_{i, d}$, is the harmonic mean SNR, $\gamma_{a, b}=\left|\alpha_{a, b}\right|^{2} E_{s} / N_{o}$ corresponds to the instantaneous SNRs of link $a-b, E_{s}$ denotes the average symbol energy and $N_{0}$ corresponds to the noise variance.

The approximation of (1) is obtained by recognizing that the instantaneous SNR of a two hops path can be accurately estimated to be the harmonic mean of the individual link SNRs, especially at moderate/high SNR regimes [43]. Hence, it is straight-forward to show that the MGF of end-toend SNRis given by

$$
\phi_{\gamma_{T}^{\text {MRC }}}(s)=\phi_{\gamma_{s, d}}(s) \prod_{i=1}^{N} \phi_{\gamma_{i}}(s)
$$

where $\phi_{\gamma_{s, d}}(s)$ and $\phi_{\gamma_{i}}(s)$ are the MGFs of the SNR for the $S$-D link and the relayed paths, respectively. The MGF of SNR for single channel reception is readily available in the literature. It has been shown in the literature that the evaluation of the MGF, PDF and CDF of $\gamma_{i}$ is a daunting task with existing results limited to Rayleigh fading [41] and Nakagami-m [42] fading channels with integer $m$ and even in such cases the expressions are too complicated, and mostly useless for the system level analysis. However, it has been shown in [43] that, $\gamma_{i}^{(H M)}$ in (1) can effectively approximate $\gamma_{i}$ especially at medium and high SNRs. Also, in this case the MGF expressions are still difficult to obtain with the existing results limited to Raleigh fading [44] and i.i.d. Nakagami$\mathrm{m}$ [43] channels. Due to this limitation, the bounds have been developed for $\gamma_{i}$ and it is given by $\gamma_{i}^{(U B)}=\min \left(\gamma_{s, i}, \gamma_{i, d}\right)$.

For instance, the closed-form formula for the MGF of $\gamma_{i}^{(U B)}$ in a Nakagami channel with i.n.d fading statistics is given by [51]

$$
\phi_{n}^{(U)}(s)=\sum_{\substack{k \in(s, i)(i, d) ! \\ j \neq k}} \frac{\Gamma\left(m_{k}+m_{j}\right)}{m_{k} \Gamma\left(m_{k}\right) \Gamma\left(m_{j}\right)}\left(\frac{\Omega_{j} m_{k}}{s \Omega_{j} \Omega_{k}+\Omega_{j} m_{k}+\Omega_{k} m_{j}}\right)^{m_{k}}{ }_{2} F_{1}\left(1-m_{j}, m_{k} ; 1+m_{k} ; \frac{\left(s \Omega_{k}+m_{k}\right) \Omega_{j}}{s \Omega_{j} \Omega_{k}+\Omega_{j} m_{k}+\Omega_{k} m_{j}}\right)
$$

where $\Omega_{q}=E\left[\gamma_{q}\right]$ corresponds to the mean SNR of link $q, \Gamma($.$) is the Gamma function and m_{q}$ is the Nakagami-m fading index.

\subsubsection{Regular CoOperation: CAF Relaying With Selection Diversity Combining (SDC) AT THE DESTINATION}

In this kind of protocol implementation, the best route is selected at the destination node based on the end-to-end relay SNR. Here in the case of SDC, the channel usage per source transmission is similar to the MRC case (i.e., $U(N)=N+1$ ).

For this protocol, the effective SNR at the output of the SDC detector (i.e., all the signals received during Phase I and Phase II) is given by

$\gamma_{T}^{S D C}=\max \left(\gamma_{s, d}, \gamma_{1}, \gamma_{2}, \ldots \gamma_{N}\right) \approx \max \left(\gamma_{s, d}, \min \left(\gamma_{s, 1} \gamma_{1, d}\right), \min \left(\gamma_{s, 2} \gamma_{2, d}\right), \ldots \min \left(\gamma_{s, N} \gamma_{N, d}\right)\right)$

For instance, the MGF of $\gamma_{T}^{S D C}$ for a special case of independent and identically distributed (i.i.d) Nakagami-m fading statistics can be obtained from Appendix A as [45] 


$$
\phi_{\gamma_{T}^{S D C}}(s) \approx\left(1+\frac{s \Omega}{m}\right)^{-m}+\sum_{p=1}^{N}(-1)^{p}\left(\begin{array}{l}
N \\
p
\end{array}\right)_{i_{1}, i_{2}, \ldots i_{2} p}^{m-1} \prod_{j=1}^{2 p} \frac{\left(\frac{m}{\Omega}\right)^{i_{j}}}{i_{j} !} \frac{s\left(\frac{m}{\Omega}\right)^{m} \Gamma(\lambda+m+1)}{\Gamma(m+1)\left[s+(1+2 p) \frac{m}{\Omega}\right]^{\lambda+m+1}} \times{ }_{2} F_{1}\left(1, \lambda+m+1 ; m+1 ; \frac{\frac{m}{\Omega}}{s+(1+2 p) \frac{m}{\Omega}}\right)
$$

where ${ }_{2} F_{1}(., . ; . ;$.$) is the Gauss hypergeometric function.$

\subsubsection{CAF Relaying With Opportunistic Route Selection AND SDC (ORS-SDC) At THE SOURCE}

The (ORS-SDC) relaying protocol focuses on limiting the number of cooperating relay to one. Additionally, the choice of the appropriate route is selected by the source (assuming that the relay with the best transmission parameter is already determined during the route discovery process) or can be accomplished in a distributed fashion among the relays as proposed in [8]. Compared to the regular cooperative diversity protocol discussed above, the best route is selected at the source based on the end-to-end relay SNR. Therefore, the statistics of the best route selection is the same as the selection diversity combining at the destination as the best among $N+1$ links is being selected. However, the channel usage per source transmission in the case is $U(N)=2$ (i.e., source will broadcast the signal to all the relays and destination in first time slot; and in the second time slot, one of the best relay amplify and forward the signal to the destination). Therefore, the spectral efficiency here does not reduce with the increasing number of relays as in the case of regular cooperative diversity protocols discussed above (i.e., MRC and SDC only). Also, the amount of channel side information and the implementation complexity is highly reduced.

\subsubsection{CAF RElaying With OPPortunistic Relay SElECtion AND MRC (ORS-MRC) AT THE DESTINATION}

This protocol implementation takes advantage of the half duplex nature of the relay transmission to achieve better performance than the ORS-SDC protocol. Here, since the source transmits in the first transmission phase, and due to the broadcast nature of the wireless channel, the destination can be close enough to receive this signal before receiving the signal from the relay. This is particularly true in the distributed ORS protocol implementation proposed in [8]. Therefore, if the channel side information of both the links is available, the received signal can be combined with the MRC scheme at the destination. Note that the transmission channel usage $U(N)=2$ each source transmission, but the statistics is slightly different from the pure ORS-SDC protocol. The effective end-to-end SNR of the ORS-MRC protocol can be expressed as

$$
\gamma_{T}^{O R S-M R C}=\gamma_{s, d}+\max \left(\gamma_{1}, \gamma_{2}, \ldots \gamma_{N}\right) \approx \gamma_{s, d}+\max \left(\min \left(\gamma_{s, 1} \gamma_{1, d}\right), \min \left(\gamma_{s, 2} \gamma_{2, d}\right), \ldots \min \left(\gamma_{s, N} \gamma_{N, d}\right)\right)
$$

The MGF of $\gamma_{T}^{O R S-M R C}$ for a special case of independent and identically distributed (i.i.d) Nakagami-m fading statistics can be obtained from Appendix B as [45]

$$
\phi_{\gamma_{T}^{\text {ORS-MRC }}}(s) \approx\left(1+\frac{s \Omega}{m}\right)^{-m}\left[1+\sum_{p=1}^{N}(-1)^{p}\left(\begin{array}{c}
N \\
p
\end{array}\right) \sum_{i_{1}, i_{2}, \ldots i_{2 p}}^{m-1} \prod_{j=1}^{2 p} \frac{\left(\frac{m}{\Omega}\right)^{i_{j}}}{i_{j} !} \frac{s \Gamma(\lambda+1)}{\left(s+2 p \frac{m}{\Omega}\right)^{(\lambda+1)}}\right]
$$

Hence, by utilizing the above mentioned closed-form MGFs of the four protocol schemes, we can easily analyse and compare the performance of CAF relay networks in terms of mean achievable spectral efficiency, outage probability and average symbol error rate. 


\subsection{Cumulative Distribution Function (CDF) OF The Total End-To-End SNR}

To evaluate the various performance measures, the knowledge of the CDF of total effective SNR $\gamma_{T}$ of the CAF relay networks is required. Since the analytical CDF expression for the CAF relay networks is difficult to obtain, the alternative is to compute the CDF from the MGF expression in (2).

One of the most efficient frequency inversion method is the Abate's Fixed-Talbot method (i.e., multi-precision Laplace transform inversion) [16], viz.,

$$
F_{X}(x) \cong \frac{1}{2 Z} \phi_{X}(r) e^{r x}+\frac{r}{Z} \sum_{k=1}^{Z-1} \operatorname{Re}\left\{\frac{1+j \sigma\left(\theta_{k}\right)}{s\left(\theta_{k}\right)} e^{x s\left(\theta_{k}\right)} \phi_{X}\left(s\left(\theta_{k}\right)\right)\right\}
$$

where $r=2 Z /(5 x), \theta_{k}=k \pi / Z, s\left(\theta_{k}\right)=r \theta_{k}\left(j+\cot \left(\theta_{k}\right)\right), \sigma\left(\theta_{k}\right)=\theta_{k}+\left(\theta_{k} \cot \left(\theta_{k}\right)-1\right) \cot \left(\theta_{k}\right), \quad$ and $\quad$ positive integer $Z$ can be chosen to get the desired accuracy. Utilizing (8), we can easily compute the CDF expressions from its respective total MGF expression for the CAF relay networks.

\section{AdAPTIVE Modulation}

In this section, we develop a new analytical framework based on the marginal MGF for evaluating the ASER, mean spectral utilization efficiency and outage probability performance metrics.It will shown that the mean achievable spectral efficiency of ADR M-QAM/M-PSK and ASER of CAF relay networks with adaptive source transmission can be expressed in terms of difference of two "CDF" terms. The ADR $M$-QAM/M-PSK schemes are first explained, followed by the outage probability, the mean spectral efficiency, and the average SER analysis.

\subsection{ADR M-QAM/M-PSK SCHEMES}

In the adaptive modulation techniques, the destination node needs only to compute the total received SNR, select the appropriate transmission rate, and feedback this information to the transmitter. In context of CAF relay system, the destination node only needs to compute and convey the information on the total (effective) received SNR to the source node for it to select an appropriate transmission rate. This results into a higher mean achievable spectral efficiency without having to sacrifice the error rate performance. For this reason and more specifically due to several other practical advantages of the adaptive rate modulation, we consider both the adaptive $M$-QAM and the M-PSK digital modulation schemes in this paper to improve the performance of the CAF relay networks.

In order to simplify the analysis of the adaptive modulation, there is a need to express instantaneous error rate in desirable exponential form, similar to the one in the existing literatures [21-25]. Here, we employ the exponential-type representations of the instantaneous (SER) for the M-PSK and the M-QAM schemes and are respectively given by [40, Table II]

$$
\begin{aligned}
& P_{S} \approx a_{1} e^{-b_{1} \gamma_{S} \sin ^{2}(\pi / M)}+c_{1} e^{-2 b_{1} \gamma_{s} \sin ^{2}(\pi / M)} \\
& P_{S} \approx 2 k a_{1} e^{-\frac{3}{2} b_{1} \gamma_{s}}+\left(2 k c_{1}-\left(k a_{1}\right)^{2}\right) e^{-\frac{3 b_{1} \gamma_{s}}{M-1}}-\left(k c_{1}\right)^{2} e^{-\frac{6 b_{1} \gamma_{s}}{M-1}}-k^{2} a_{1} c_{1} e^{-\frac{9}{2} \frac{b_{1} \gamma_{s}}{M-1}}
\end{aligned}
$$


where $k=(\sqrt{M}-1) / \sqrt{M}, M$ is the constellation size and parameters $a_{1}=0.2938 ; b_{1}=1.0483$; $c_{1}=0.5070$ are obtained from [40, Table I]. The above exponential forms particularly facilitate the averaging of the SER over the fade distribution. The resulting average symbol error rate (ASER) expression (taking advantage of Laplace transform property), which can be evaluated as the weighted sum of the MGF of end-to-end SNR of fading channel, can be expressed as

$$
\begin{aligned}
& \bar{P}_{M-P S K}=a_{1} \phi_{\gamma}\left(b_{1} \sin ^{2}(\pi / M)\right)+c_{1} \phi_{\gamma}\left(2 b_{1} \sin ^{2}(\pi / M)\right) \\
& \bar{P}_{M-Q M M}=2 k a_{1} \phi\left(\frac{3 b_{1}}{2(M-1)}\right)+\left(2 k c_{1}-\left(k a_{1}\right)^{2}\right) \phi\left(\frac{3 b_{1}}{M-1}\right)-\left(k c_{1}\right)^{2} \phi\left(\frac{6 b_{1}}{M-1}\right)-k^{2} a_{1} c_{1} \phi\left(\frac{9 b_{1}}{2(M-1)}\right)
\end{aligned}
$$

where $\phi_{\gamma}($.$) is the MGF of SNR for single channel.$

In the ADR M-QAM/M-PSK system, the range of the effective received SNR is divided into $T+1$ fading regions. When the fading causes the total received SNR to fall into the $n$-th region $(n=0$, $1, T)$, the constellation size $M_{n}=2^{n}$ is employed for the transmission. Also, the SNR thresholds for partitioning of the total received SNR depends on the target SER level, $P_{s}$. The region boundary $\gamma_{n}$ is chosen for the corresponding transmission mode $n$ to be the minimum SNR required to achieve $P_{s}$, which can be easily obtained by inverting (9) and (10) for M-PSK and MQAM modulation schemes respectively

$$
\begin{aligned}
& \gamma_{n} \approx-\frac{1}{b_{1} \sin ^{2}\left(\pi / M_{n}\right)} \ln \left(\frac{-a_{1}+\sqrt{a_{1}^{2}+4 c_{1} P_{S}}}{2 c_{1}}\right) \\
& \gamma_{n} \approx-\frac{2\left(M_{n}-1\right)}{3 b_{1}} \ln \left(\frac{-a_{1}+\sqrt{a_{1}^{2}+4 c_{1}\left(\frac{\left.\sqrt{M_{n}}\right)\left(1-\sqrt{1-P_{S}}\right)}{\sqrt{M_{n}-1}}\right)}}{2 c_{1}}\right)
\end{aligned}
$$

where $, n=1,2,3 \ldots, T$ and $\gamma_{T+1}=+\infty$.

It is worth to mention here that, the two exponential terms are more accurate than the existing invertible expressions in the literature [46-48]. Representative example has been shown in Fig. 2, where a comparison has been made between the proposed approximation and the single and two exponential term approximation in [47] and [48] respectively. This figure highlights that our proposed exponential approximation (11-12), yields a very good estimate of the actual ASER performance over a wide range of average link SNRs, different fading severity indices and for different constellation sizes. It is evident from the figure that, the proposed approximation performs better than [47] and [48] for the M-QAM and better than [48] for the M-PSK. Therefore, for the rest of the analysis in this article, the proposed two exponential term approximation will be utilized. 
International Journal of Computer Networks \& Communications (IJCNC) Vol.8, No.3, May 2016

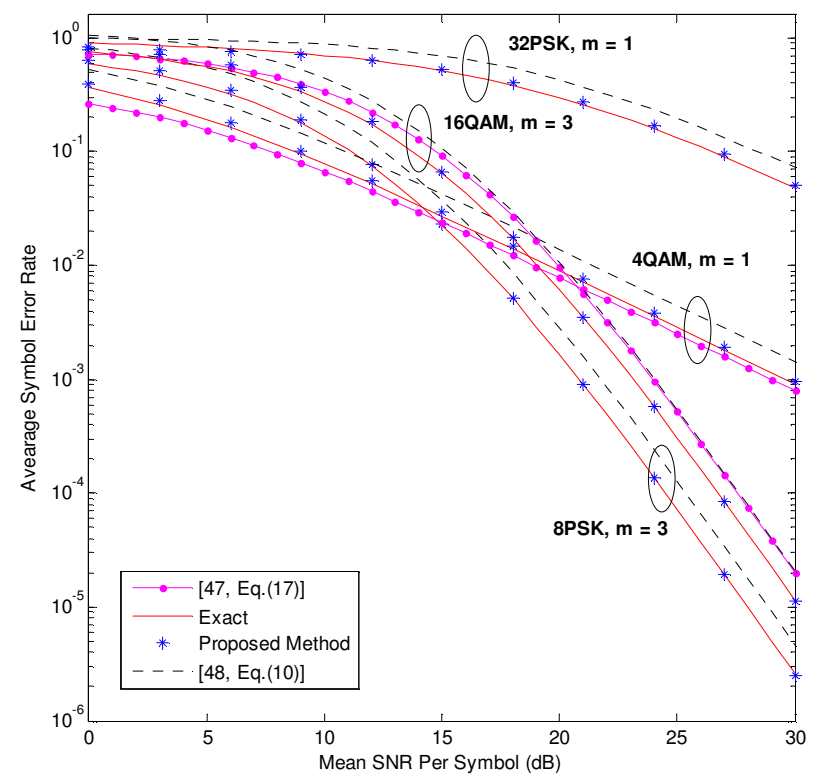

Figure 2 ASER of M-PSK and M-QAM over Nakagami fading channels ( $m=1$ and 3 ) without diversity

\subsection{Outage Probability}

When the total received SNR falls below the region boundary threshold $\gamma_{1}$ ( $\gamma_{1}$ can be obtained by setting, $n=1$ in (13) or (14)), we cease the transmission, because the prescribed target SER cannot be satisfied even with the smallest constellation size. The probability of such an outage event is given by $P_{\text {out }}=F_{\gamma}\left(\gamma_{1}\right)$, where the CDF term can be evaluated efficiently using (8).

\subsection{MeAn SPECTRAL EFFiCIENCY}

The normalized average achievable spectral efficiency for ADR M-QAM/PSK is given by the weighted sum of the data rates in each of the partitioned regions [21, 25] viz.,

$\frac{R_{a d r}}{B}=\frac{1}{U(N)} \sum_{n=1}^{T} n p_{n}$

where $p_{n}$ denotes the transmission mode selection probability (i.e., probability that the total received SNR falls in the $n$-th partition region):

$p_{n}=\int_{\gamma_{n}}^{\gamma_{n+1}} f_{\gamma}(\gamma) d \gamma=F_{\gamma}\left(\gamma_{n+1}\right)-F_{\gamma}\left(\gamma_{n}\right)$

Hence, using the appropriate MGF expressions derived in [43-44, 49] in (8), we can readily compute the mean spectral efficiency of the ADR M-QAM/PSK in a myriad of wireless fading environments. 


\subsection{ASER OF ADAPTIVE M-QAM/PSK}

The ASER of ADR M-QAM/PSK can be calculated as the ratio of the average number of error bits per transmission divided by the average number of bits per transmission [47], viz.,

$$
\operatorname{ASER}_{a d r}=\frac{\sum_{n=1}^{T} n \overline{\operatorname{SER}}}{\sum_{n=1}^{T} n\left[F_{\gamma}\left(\gamma_{n+1}\right)-F_{\gamma}\left(\gamma_{n}\right)\right]},
$$

where $\overline{S E R_{n}}$ is the average SER in a specific SNR region of $\left[\gamma_{n+1}, \gamma_{n}\right]$ and can be represented as

$$
\overline{S E R_{n}}=\int_{\gamma_{n}}^{\gamma_{n+1}} P_{M} f_{\gamma}(\gamma) d \gamma
$$

where $P_{M}$ is the CEP of the modulation scheme in AWGN channel. For the special case of MPSK scheme, we can derive the $\overline{S E R_{n}}$ by substituting (9) in to (18) viz.,

$$
\begin{aligned}
\overline{\operatorname{SER}_{n}} & =\int_{\gamma_{n}}^{\gamma_{n+1}}\left\{a_{1} \exp \left(-b_{1} \gamma \sin ^{2}(\pi / M)\right)+c_{1} \exp \left(-2 b_{1} \gamma \sin ^{2}(\pi / M)\right)\right\} f_{\gamma}(\gamma) d \gamma \\
& =a_{1}\left[\phi_{\gamma}\left(b_{1} \sin ^{2}(\pi / M), \gamma_{n+1}\right)-\phi_{\gamma}\left(b_{1} \sin ^{2}(\pi / M), \gamma_{n}\right)\right]+c_{1}\left[\phi_{\gamma}\left(2 b_{1} \sin ^{2}(\pi / M), \gamma_{n+1}\right)-\phi_{\gamma}\left(2 b_{1} \sin ^{2}(\pi / M), \gamma_{n}\right)\right]
\end{aligned}
$$

and the term $\phi_{\gamma}(\beta, \alpha)=\int_{\alpha}^{\infty} e^{-\beta \gamma} f_{\gamma}(\gamma) d \gamma$ in (19) denotes the marginal MGF of the total received SNR.

Note that although this quantity is available in closed-form for non-cooperative system (e.g. [48]), similar expressions do not appear to be readily available or generalized for the CAF relay networks, particularly in a generalized wireless fading environment. Utilizing [51, Appendix C], we can compute the desired marginal MGF as a difference between two "CDF" terms of an auxiliary function, viz.,

$$
\overline{S E R_{n}}=a_{1}\left[F_{\hat{\gamma}_{a}}\left(\gamma_{n+1}\right)-F_{\hat{\gamma}_{a}}\left(\gamma_{n}\right)\right]+c_{1}\left[F_{\hat{\gamma}_{b}}\left(\gamma_{n+1}\right)-F_{\hat{\gamma}_{b}}\left(\gamma_{n}\right)\right]
$$

where $F_{\hat{\gamma}_{a}}(x)$ and $F_{\hat{\gamma}_{b}}(x)$ in (20) can be evaluated efficiently via (8), but using the "MGF" formulas of the auxiliary functions (i.e., $\phi_{r}(s)=\phi_{\gamma}\left(s+b_{1} \sin ^{2}(\pi / M)\right)$ and $\phi_{\gamma_{b}}(s)=\phi_{\gamma}\left(s+2 b_{1} \sin ^{2}(\pi / M)\right)$ ). Similarly treatment can also be applied to the M-QAM modulation scheme by substituting (10) into (18).

Eq. (20) allows us to generalize the evaluation of $A S E R_{\text {adr }}$ over arbitrary multipath/shadowing fading as long as the MGF of SNR of fading channel is available. This is in sharp contrast with channel specific PDF methods in [32-34] which limited their analysis to Rayleigh fading channel.

\section{NUMERICAL RESUltS}

In this section, selected numerical results are provided for the normalized mean achievable spectral efficiency, outage probability and ASER performance metrics of CAF relay networks with both the adaptive discrete rate M-QAM and M-PSK digital modulation schemes. In particular, we are comparing the performance of four distinct cooperative diversity and 
opportunistic routing protocols: (i) ORS-MRC (ii) ORS-SDC (iii) MRC and (iv) SDC, over the i.i.d Nakagami fading environment (including special case of Rayleigh fading). Moreover, the ergodic capacity analysis with the optimal rate adaptation policy is also presented for the theoretical performance limit of the above protocol schemes. To generate plots, the mean link SNRs are chosen arbitrarily as: $\Omega_{s, 1}=\Omega_{s, 2}=\Omega_{s, 3}=\Omega_{1, d}=\Omega_{2, d}=\Omega_{s, d}=\Omega_{s, d}=E_{s} / N_{0}$ and the fading indexes on the each links is chosen as $m=3$ (unless states otherwise). For the ADR system, the target SER of $10^{-3}$ is arbitrarily chosen.

Fig. 3 illustrates the ergodic capacities of the ORA policy using four different protocols (i.e., ORS-MRC, ORS-SDC, SDC and MRC). To generate the plots, we have used the following generalized expressions in terms of the MGF of end-to-end SNR of CAF relay networks [11]

$$
\frac{\bar{C}_{O R A}}{B}=\frac{1}{U(N)} \frac{1}{\ln 2} \int_{0}^{\infty} \frac{e^{-y}}{y}\left[1-\phi_{\gamma}(y)\right] d y
$$

Expression (21) indicates that the ORA capacity evaluation requires only the knowledge of the MGF of SNR of the fading channel. By substituting the total MGF of the above mentioned protocols into (21), we can easily generate the curves as shown in the figure. From the figure, we can observe that the performance of the opportunistic relay scheme (i.e., ORS-MRC and ORSSDC) is better than the regular cooperation (i.e., MRC and SDC) respectively. This improvement in the performance of the ORS scheme is due to the utilization of the two orthogonal slots for the total transmissions compared to the three slots in the regular cooperation. Moreover, it is interesting to note that, the authors in [28], compares the ergodic capacities of the CAF relay network using best relay selection and the regular MRC scheme. However, their framework does not lend itself to the analysis of the ORS-SDC or the SDC case, whereas our framework encapsulates the performance of all the four protocols.

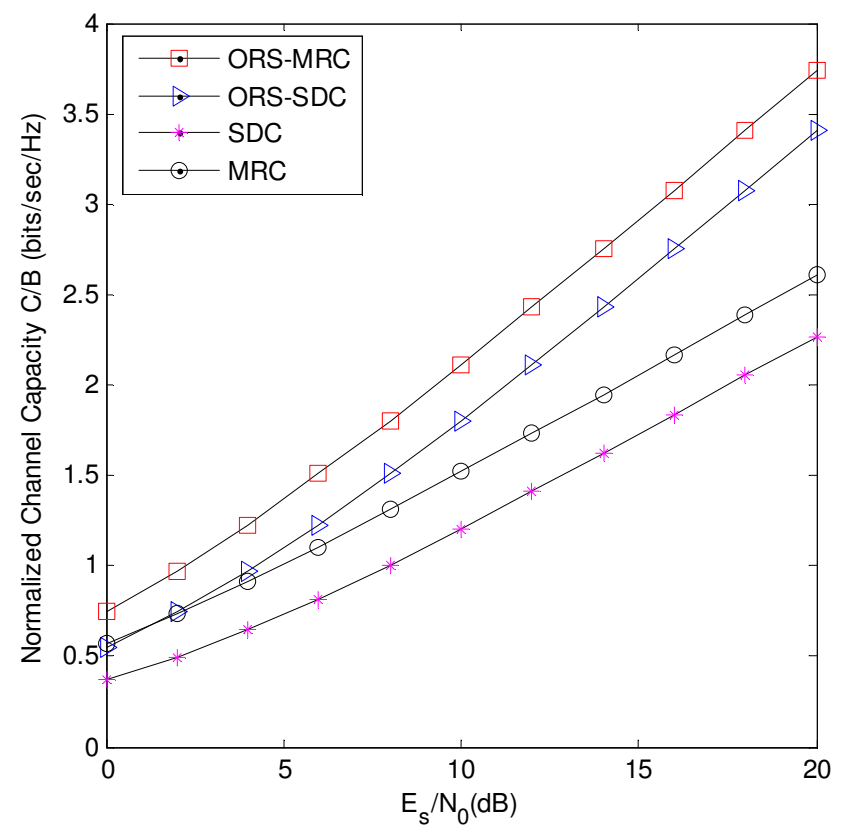

Figure 3 Ergodic channel capacities of the optimal rate adaptation (ORA) policy with two cooperative relays $(\mathrm{N}=2)$ 
Fig. 4 shows the comparison in terms of spectral efficiency of the different cooperative diversity and the opportunistic routing protocols, with the adaptive M-PSK modulation. It is worth to mention that for a single relay case, the ORS-MRC gives the same performance as the MRC scheme, while the ORS-SDC scheme also gives the same performance as the SDC scheme.

However, as the number of relay increases, ORS-MRC performs better than the regular MRC protocol at medium and high SNRs, while the ORS-SDC protocol performs better than the regular SDC protocol throughout the SNR range. This is because irrespective of the number of relays in the participation, the total channel usage for the ORS-MRC and the ORS-SDC is kept constant at two time slots per source transmission; whereas the channel usage for MRC and SDC schemes increases with increasing number of relays. Moreover, to further improve the spectral efficiency, we incorporate the adaptive M-PSK modulation scheme (compared to fixed modulation schemes in the previous literatures) to adapt the transmission rate with the varying channel conditions. It is evident from Fig. 4 that by increasing the maximum constellation size (transmission modes) in the ADR M-PSK directly translates into improved spectral efficiency. However, this improvement is achieved at the expense of the increased ASER (see Fig. 5). In summary, the link adaptive ORS-MRC scheme gives the best overall performance. Hence, it can be concluded that the ORS-MRC protocols are recommended for the cooperating nodes at the tactical edge or at the cell boundary, where the received signal strength is weak.

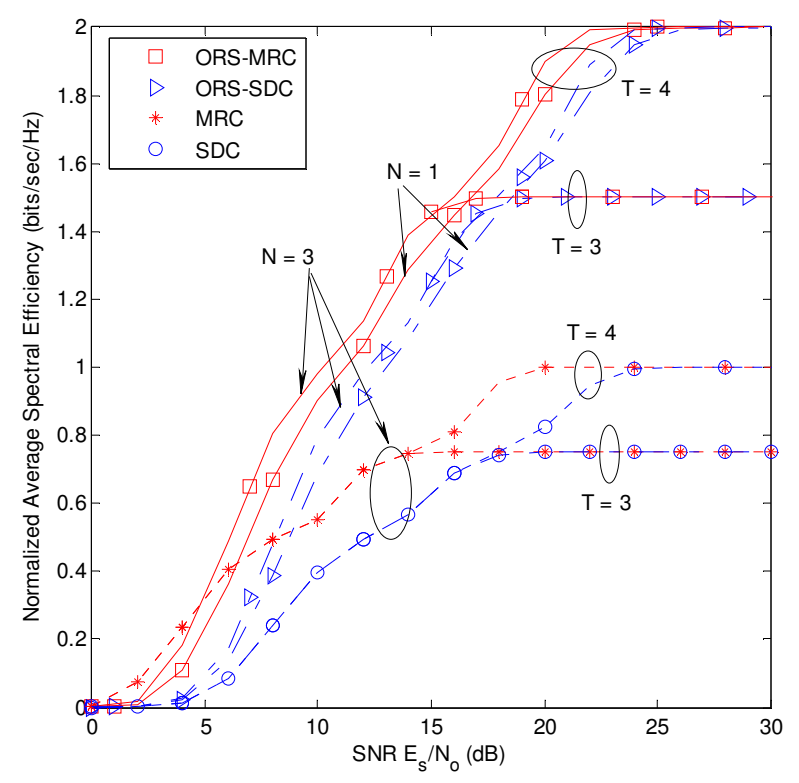

Figure 4 Comparison of different cooperative diversity and opportunistic routing protocols with adaptive M-PSK modulation ( $\mathrm{T}=3$ and 4$)$

Fig. 5 illustrates the average symbol error rate (ASER) of a CAF relay network with the adaptive M-PSK modulation (using $T=3$ and 4). We observe that the ASER of the MRC scheme is the lowest, whereas, the ORS-SDC scheme is the highest. This is due to the availability of the total $N+1$ diversity paths in the MRC scheme. However, this is achieved at the expense of the power efficiency as the ORS-SDC and the ORS-MRC requires only $1 /(N+1)$ and $2 /(N+1)$ of the total power of the regular MRC scheme respectively. 
International Journal of Computer Networks \& Communications (IJCNC) Vol.8, No.3, May 2016

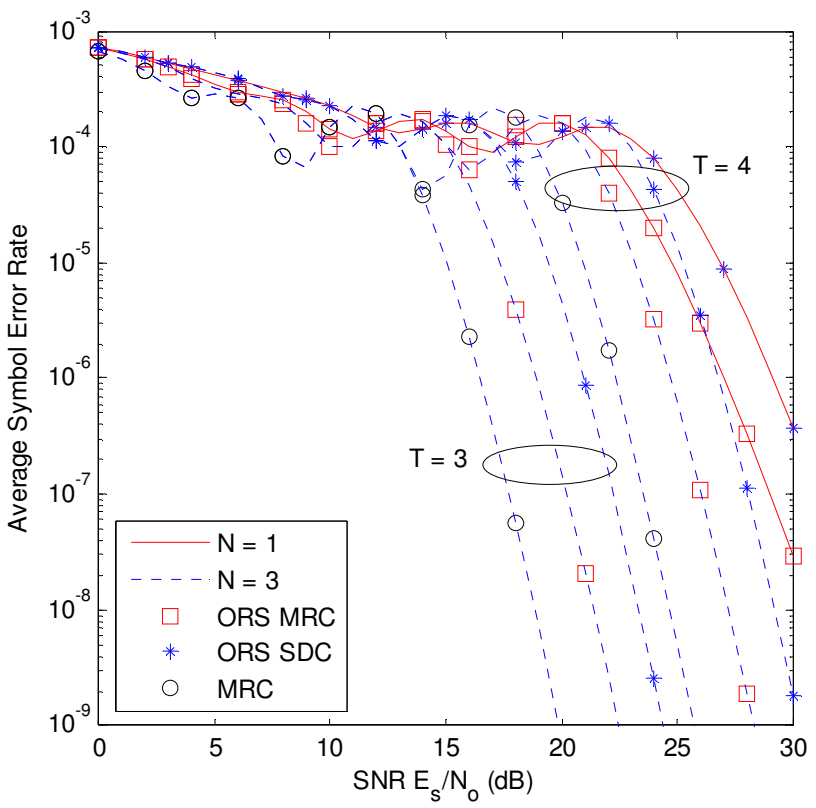

Figure 5 Average symbol error rate of a CAF system with different cooperative diversity and opportunistic routing protocols using adaptive M-PSK modulation ( $T=3$ and 4$)$

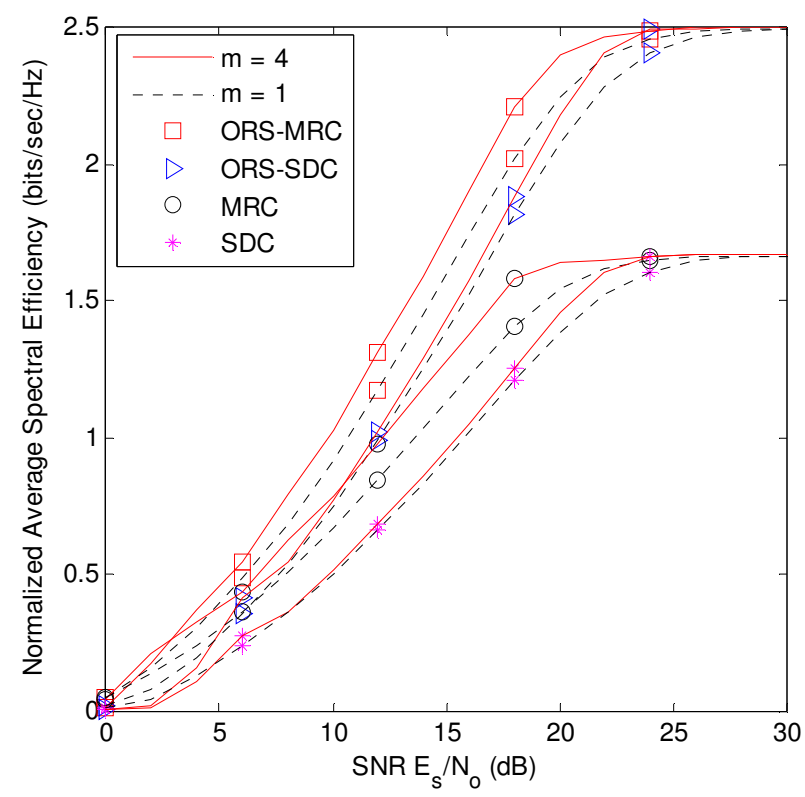

Figure 6 Comparison of different cooperative diversity and opportunistic routing protocols with adaptive M-MQAM modulation $(\mathrm{T}=5)$ consisting of two relays

Fig. 6 shows the spectral efficiency performance comparison of the four cooperative diversity and the opportunistic routing protocols with the ADR M-QAM modulation scheme with $T=5$. This figure highlights the influence of the channel fading severity on the performance of the link adaptive cooperative system. While the performance trend among the protocols is similar to the one obtained in Fig. 4, Fig. 6 in particularly shows that, as the channel condition improves, the 
achievable spectra efficiency improves. To the best of our knowledge, this effect has never been reported in the earlier literature, and it also demonstrates the versatility of our mathematical framework.

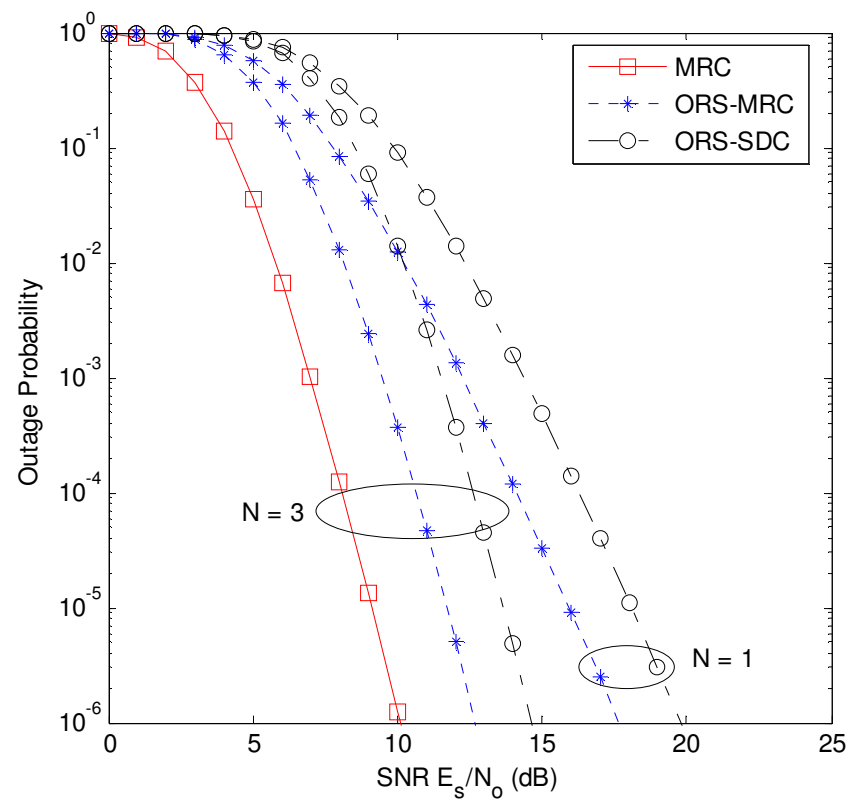

Figure 7 Probability of outage of a CAF system with different cooperative diversity and opportunistic routing protocols using adaptive M-PSK modulation $(\mathrm{T}=3$ ) (Note that for $\mathrm{N}=1$, the ORS-MRC and MRC, whereas for any values of N, ORS-SDC and SDC schemes are the same)

Figure 7 depicts the outage probability as a function of SNR at the target SER of $10^{-3}$ and it highlights the benefit of the cooperative diversity to maximize the performance of the wireless communication system. From figure 7, we conclude the following important observations. First, we notice that the case with cooperative diversity (i.e., $N=3$ ) evidently outperforms the case with $N=1$. Second, the outage probability with the MRC protocol has a better performance than all the other protocols (similar to the case in fig. 5 for the ASER analysis with the MRC protocol). This performance gain is due to the additional diversity path offered by all the relays and direct path in the system, but still at the expense of the power efficiency.

\section{Conclusions}

This paper analyzes the performance of cooperative amplify-and-forward (CAF) relay networks that employ the adaptive M-ary quadrature amplitude modulation (M-QAM)/M-ary phase shift keying (M-PSK) digital modulation techniques in the Nakagami-m fading channel model. In particular, we present and compared the analysis of the CAF relay networks with different cooperative diversity and opportunistic routing protocols such as Maximal Ratio Combining (MRC), Selection Diversity Combining (SDC), Opportunistic Relay Selection with Maximal Ratio Combining (ORS-MRC) and Opportunistic Relay Selection with Selection Diversity Combining (ORS-SDC).We advocate a simple yet unified numerical approach based on the marginal moment generating function (MGF) of the total received SNR to compute the average symbol error rate (ASER), mean achievable spectral efficiency, and the outage probability performance metrics. These analytical frameworks and results will facilitate the choice of cooperation protocol and configurations that can be employed in the design and deployment of femtocells. 
International Journal of Computer Networks \& Communications (IJCNC) Vol.8, No.3, May 2016

\section{APPENDix A}

This section provides the derivation for the MGF of end-to-end SNR of CAF relay system with SDC protocol at the destination. This is also applicable to the ORS scheme with SDC at the destination.

The CDF of the end-to-end SNR given in (4) can be expressed as

$$
F_{\gamma_{T}^{S D C}}(\gamma)=F_{\gamma_{s, d}}(\gamma) \prod_{r=1}^{N} F_{\gamma_{r}}(\gamma) \approx F_{\gamma_{s, d}}(\gamma) \prod_{r=1}^{N}\left(1-\left[1-F_{\gamma_{s, r}}(\gamma)\right]\left[1-F_{\gamma_{r, d}}(\gamma)\right]\right)
$$

where $F_{\gamma_{s, d}}(\gamma), F_{\gamma_{s, r}}(\gamma)$ and $F_{\gamma_{r, d}}(\gamma)$ are the CDFs of the source-to-destination, source-to-relay and relay-to-destination links, respectively.

The effective MGF can then be evaluate using the differentiation property of the Laplace transform via a single integral expression

$$
\begin{aligned}
\phi_{\gamma_{T}^{S D C}}(s) & =s \int_{0}^{\infty} e^{-s \gamma} F_{\gamma_{s, d}}(\gamma) \prod_{r=1}^{N} F_{\gamma_{r}}(\gamma) d \gamma \\
& \approx s \int_{0}^{\infty} e^{-s \gamma} F_{\gamma_{s, d}}(\gamma) \prod_{r=1}^{N}\left(1-\left[1-F_{\gamma_{s, r}}(\gamma)\right]\left[1-F_{\gamma_{r, d}}(\gamma)\right]\right) d \gamma
\end{aligned}
$$

For special case of independent and identically distributed (i.i.d.) Nakagami- $m$ channel, the MGF can be reduced to [30]

$$
\phi_{\gamma_{T}^{S D C}}(s) \approx s \int_{0}^{\infty} e^{-s \gamma} \frac{G\left(m, \frac{m}{\Omega} \gamma\right)}{\Gamma(m)}\left[1+\sum_{p=1}^{N}(-1)^{p}\left(\begin{array}{c}
N \\
p
\end{array}\right) e^{-\left(2 p \frac{m}{\Omega}\right) \gamma} \sum_{i_{1}, i_{2}, \ldots i_{2 p}}^{m-1} \gamma^{\lambda} \prod_{j=1}^{2 p} \frac{\left(\frac{m}{\Omega}\right)^{i_{j}}}{i_{j} !}\right] d \gamma_{(\mathrm{A} .3)}
$$

where $G(.,$.$) is the lower incomplete gamma function and \lambda=\sum_{j=1}^{2 p} i_{j}$

Using the identity [52, Eq. (6.455.2)], after few algebraic manipulations, the closed-form MGF expression can be obtained as shown in (5).

\section{APPENDIX B}

This section provides the derivation for the MGF of end-to-end SNR of ORS CAF relay system with MRC protocol at the destination.

The effective MGF of (6) can be evaluated using the addition and differentiation properties of the Laplace transform via a single integral expression given by

$$
\begin{aligned}
\phi_{\gamma_{T}^{O R S-M R C}}(s) & =s \phi_{\gamma_{s, d}}(s) \int_{0}^{\infty} e^{-s \gamma} \prod_{r=1}^{N} F_{\gamma_{r}}(\gamma) d \gamma \\
& \approx s \phi_{\gamma_{s, d}}(s) \int_{0}^{\infty} e^{-s \gamma} \prod_{r=1}^{N}\left(1-\left[1-F_{\gamma_{s, r}}(\gamma)\right]\left[1-F_{\gamma_{r, d}}(\gamma)\right]\right) d \gamma
\end{aligned}
$$


For special case of independent and identically distributed (i.i.d.) Nakagami- $m$ channel, the MGF can be reduced to

$$
\phi_{\gamma_{T}^{\text {ORS-MRC }}}(s) \approx\left(1+\frac{s \Omega}{m}\right)^{-m}\left[1+\sum_{p=1}^{N}(-1)^{p}\left(\begin{array}{c}
N \\
p
\end{array}\right) \sum_{i_{1}, i_{2}, \ldots i_{2}}^{m-1} \prod_{j=1}^{2 p} \frac{\left(\frac{m}{\Omega}\right)^{i_{j}}}{i_{j} !} s \int_{0}^{\infty} \gamma^{\lambda} e^{-s \gamma} e^{-\left(2 p \frac{m}{\Omega}\right) \gamma} d \gamma\right]
$$

Using the identity [52, Table 17.13], after few algebraic manipulations, the closed-form MGF expression can be obtained as expressed in (7).

It is worth to mention that the expression in (7) is much more compact and simpler than the equivalent expression in [30, Eq. (17)].

\section{ACKNOWLEDGMENT}

This work is supported in part by funding from the National Science Foundation NSF/HRD 0931679 and the US Air Force Research Laboratory (Contract \#FA8750-09-1-0151).

\section{REFERENCES}

[1] Cisco Visual Networking Index: Forecast and Methodology,20102015.http://www.cisco.com/en/US/solutions/collateral/ns341/ns525/ns537/ns705/ns827/white_paper_ c11-481360_ns827_Networking_Solutions_White_Paper.html. Accessed February 01, 2011

[2] ITU global standard for international mobile telecommunications 'IMT-Advanced'. http://www.itu.int/ITU-R/index.asp?category=information\&rlink=imt- advanced \& lang=en. Accessed February 01, 2011

[3] N. Laneman, D. Tse, and G. Wornell, (2004) "Cooperative diversity in wireless networks: efficient protocols and outage behavior," IEEE Trans. Info. Theory, vol. 50, no.12, pp. 3062-3080.

[4] N. Lanemanand G. Wornell, (2003) "Distributed space-time coded protocols for exploiting cooperative diversity in wireless networks. IEEE Trans. Info. Theory, vol. 49, no .10, pp. 2415-2525.

[5] A. Sendonaris, E. Erkip and B. Aazhang, (2003) "User cooperation diversity, part I: system description”IEEE Trans. Communications, vol. 51, no.11, pp. 1927-1938.

[6] A. Sendonaris, E. Erkip and B. Aazhang,(2003) "User cooperation diversity, part II: implementation aspects and performance analysis",IEEE Trans. Communications, vol. 51, no. 11, pp. 1939-1948.

[7] M. Khojastepour, A. Sabharwal and B. Aazhang, (2004) "Lower bounds on the capacity of Gaussian relay channel",Conf. Information Sciences and Systems (CISS), Princeton, NJ, pp. 597-602.

[8] A. Bletsas, A. Khisti,D. P. Reedand A. Lippman, (2006) "A simple cooperative diversity method based on network path selection,'IEEE Journal on Selected Areas on Communications, vol. 24, no.3, pp. 659-672.

[9] Y. Zhao, R. Adve, and T. J. Lim, (2006) "Improving amplify-and-forward relay networks: optimal power allocation versus selection”,IEEE International Symposium on Information Theory, pp. 12341238.

[10] Y. Zhao, R. Adve and T. J. Lim, (2006) "Symbol error rate of selection amplify-and-forward relay systems",IEEE Communications Letters, vol. 10, no. 11, pp. 757-759.

[11] A. Bletsas, H. Shin, and M. Z. Win, (2007) "Cooperative communications with outage-optimal opportunistic relaying”, IEEE Transactions on Wireless Communications, vol. 6, no. 9, pp. 1-11.

[12] B. Modi, A. Annamalai, O. Olabiyi and R. Palat, (2013) "Ergodic capacity analysis of cooperative amplify-and-forward relay networks over generalized fading channels",Wiley Journal of Wireless and Mobile Computing, vol. 15, no. 8, pp.1259-1273

[13] T. Nechiporenko, K. Phan, C. Tellambura and H. Nguyen, (2009) "Capacity of Rayleigh fading cooperative systems under adaptive transmission”, IEEE Trans. Wireless Comm., vol. 8, no.4, pp. 1626-1631. 
[14] A. Annamalai, R. Palat and J. Matyjas, (2010) "Estimating ergodic capacity of cooperative analog relaying under different adaptive source transmission techniques", IEEE Sarnoff Symposium, pp. 1-4.

[15] A. Annamalai, B. Modi, R. Palat and J. Matyjas, (2010) "Tight-bounds on the ergodic capacity of cooperative analog relaying with adaptive source transmission techniques",IEEE International Symposium on Personal, Indoor, and Mobile Radio Comm., pp. 18-23.

[16] R. Palat, A. Annamalai and J. Reed, (2008) "An efficient method for evaluating information outage probability and ergodic capacity of OSTBC systems”, IEEE Comm. Letters, vol. 12, no.3, pp. 191193.

[17] M. Di. Renzo, F. Graziosi, and F. Santucci, (2010) "Channel capacity over generalized fading channels: A novel MGF-based approach for performance analysis and design of wireless communication systems", IEEE Trans. Vehicular Technology, vol. 59, no.1, pp. 127-149.

[18] B. Modi, O. Olabiyi, A. Annamalai and D. Vaman, (2011)"On ergodic capacity of cooperative nonregenerative relay networks in Rice fading environments”, IEEE GLOBECOMWorkshop, pp. 348352

[19] B. Modi, O. Olabiyi, A. Annamalai and D. Vaman, (2011) "Improving the spectral efficiency of adaptive modulation in amplify-and-forward cooperative relay networks with an adaptive ARQ protocol”, IEEE Global Telecommunications Conference, pp. 1-5

[20] M. Hasna, (2005) "On the capacity of cooperative diversity systems with adaptive modulation", International Conference on Wireless and Optical Communication Networks, pp. 432-436.

[21] T. Nechiporenko, K. Phan, C. Tellambura and H. Nguyen, (2008) "Performance analysis of adaptive M-QAM for Rayleigh fading cooperative systems", IEEE International Conference on Communications, pp. 3393-3399.

[22] T. Nechiporenko, P. Kalansuriya and C. Tellambura, (2009) "Performance of optimum switching adaptive M-QAM for amplify-and-forward relays," IEEE Trans. Vehic. Tech., vol. 58, no. 5, pp. 2258-2268.

[23] K. Hwang, Y. Ko and M. Alouini, (2008) "Performance analysis of opportunistic incremental relaying with adaptive modulation over cooperative networks", IEEE International Symp. Wireless Pervasive Comput.,pp. 586-590.

[24] P. Kalansuriya and C. Tellambura, (2009) "Performance analysis of decode-and-forward relay network under adaptive M-QAM”, IEEE International Conference on Communications, pp. 33933399.

[25] B. Modi and A. Annamalai, (2011) "Improving the spectral efficiency of amplify-and-forward cooperative relay network with adaptive M-QAM modulation”, 20th IEEE International Conference on Computer Communications and Networks, pp. 1-6.

[26] B. Barua, N. Quoc and H. Shin, (2008) "On the SEP of cooperative diversity with opportunistic relaying”, IEEE communication letters, vol. 12, no. 10, pp. 727-729.

[27] S. Ikki, and M. H. Ahmed, (2008) "Performance of multiple-relay cooperative diversity system with best relay selection over Rayleigh fading channels”,EURASIP Journal on Advances on Signal Processing, article ID 580368.

[28] S. I. Hussain, M.S. Alouini and M. O. Hasna, (2010) "Performance analysis of best relay selection scheme for amplify-and-forward cooperative networks in identical Nakagami-m channels",IEEE Signal Processing Advances in Wireless Communications Conference, pp. 1-5.

[29] S. I. Hussain, M.O Hasna and M.S. Alouini, (2010)"Performance analysis of best relay selection scheme for fixed gain cooperative networks in non-identical Nakagami-m channels", IEEESignal Processing Advances in Wireless Communications Conference, pp. 255-259.

[30] O. Waqar, D. C. McLernon and M. Ghogho (2009) "Performance analysis of non-regenerative opportunistic relaying in nakagami-m fading",IEEE International Symposium on Personal, Indoor, and Mobile Radio Comm., pp. $231-235$.

[31] S. Valentin, S. I. Hermann, H. Karl, L. Loyola and J. Widmer, (2008) "Opportunistic relaying vs. selective cooperation: analyzing the occurance-conditioned outage capacity",11th International Symposium on Modeling Analysis and Simulation of Wireless and Mobile Systems, pp. 193-202.

[32] S. Ikki, and M. H. Ahmed, (2010) "Performance analysis of adaptive decode-and-forward cooperative diversity networks with best relay selection'IEEE Transactions on Communications, vol. 58, no. 1, pp. 68-72.

[33] S.Ikki, and M. H. Ahmed, (2010) "On the capacity of relay selection cooperative diversity networks under adaptive transmission”, IEEE Vehicular Technology Conf., pp. 1-5. 
International Journal of Computer Networks \& Communications (IJCNC) Vol.8, No.3, May 2016

[34] M. Torabi, andD. Heccoun, (2010) "Capacity analysis of opportunistic relaying in cooperative systems with outdated channel information”,IEEE Communication Letters, vol. 14,no.12, pp. 1-3.

[35] M. Torabi, J. F. Frigon and D. Haccoun, (2011) "On the performance analysis of AF opportunistic relaying with adaptive transmission over Rayleigh fading channels", IEEE pacific Rim conference on communications, computers and signal processing, pp. 173-178.

[36] E. S. Altubaishi, and X. S. Shen, (2011) "Spectrally efficient variable-rate best-relay selection scheme for adaptive cooperative system", IEEE GLOBECOM Conference, pp. 1-5.

[37] M. Torabi, J.F. Frigon, and D. Haccoun, (2011) "Performance analysis of variable rate adaptive modulation for AF opportunistic relaying under outdated CSI" IEEEInternational Symposium on Personal, Indoor and Mobile Radio Communication, pp. 1753-1757

[38] K.S. Hwang, Y.C. Ko, and M.S. Alouini, (2009) "Performance analysis of incremental opportunistic relaying over identically and non-identically distributed cooperative paths", IEEE Transactions on Wireless Communications, vol. 8, no. 4, pp. 1953-1960.

[39] H.Y. Lateef, D. C. McLermon and M. Ghogho, (2010) "Performance analysis of cooperative communication with opportunistic relaying", IEEE 11thInternational Workshop on Signal Processing Advances in Wireless Communications, pp. 1-5

[40] O. Olabiyi and A. Annamalai, (2011) "ASER analysis of cooperative non-regenerative relay systems over generalized fading channels", IEEE International Conference on Computer Communication and Networks, pp. 1-6.

[41] R. H. Y. Louie, Y. Li, and B. Vucetic, (2008) "Performance analysis of beamforming in two hop amplify and forward relay networks",IEEE International Conference on Communications, pp. 43114315 .

[42] D. Senarante and C. Tellambura, (2010)"Unified exact performance analysis of two-hop amplify-andforward relaying in Nakagami fading”, IEEE Trans. Veh. Tech., vol. 59, no.3, pp.1529-1534.

[43] M. Hasna and M.S. Alouini (2004) "Harmonic mean and end-to-end performance of transmission system with relays", IEEE Trans. Communications, vol. 52, no 1, pp. 130-135.

[44] Weifeng Su, K. S. Ahmed andK. J. Ray Liu, (2008) "Cooperative communication protocols in wireless networks: performance analysis and optimum power allocation”, Springer link Wireless Personal Communication, vol. 44, no. 2, pp.181-217.

[45] O. Olabiyi, A. Annamalai, O. Odejideand E. Adebola, (2012)"Integrated design of APP/NET/PHY/MAC layers for cooperative relay networks. Under review for Publication, International Journal of Wireless and Mobile Computing

[46] O. Olabiyi and A. Annamalai, (2012) "Efficient symbol error rate analysis of cooperative nonregenerative relay systems over generalized fading channels",International Journal of Wireless and Mobile Networks, vol. 4, no.1, pp. 1-20

[47] A. Goldsmith and S. Chua, (1997) "Variable-rate variable-power M-QAM for fading channels", IEEE Trans. Commun., vol. 45, no. 10, pp. 1218-1230.

[48] M. Chiani, D. Dardari and M. K. Simon, (2003) "New exponential bounds and approximations for the computation of error probability in fading channels", IEEE Trans. On Wireless Commun., vol. 2, no. 4, pp. 840-845.

[49] M. Di. Renzo, F. Graziosi and F. Santucci, (2009) "A unified framework for performance analysis of CSI-assisted cooperative communications over fading channels”,IEEE Trans. Communications, vol. 57, pp. 2551-2557.

[50] A. Annamalai, G.Deora and C. Tellambura, (2005) "Theoretical diversity improvement in GSC (N, L) receiver with non identical fading statistics”, IEEE Trans. Commun., vol. 53, no. 6, pp. 10271035.

[51] B. Modi, A. Annamalai, O. Olabiyi and R. Chembil Palat, (2012) "Ergodic capacity analyses of cooperative amplify and forward relay networks over Rice and Nakagami fading channels", International Journal of Wireless and Mobile Networks, vol. 4, no.1, pp. 97-116.

[52] I. S. Gradshteyn and I. M. Ryzhik, Table of Integrals, Series and Products, 5th ed., San Diego, CA: Academic, 1994. 


\section{AuTHORS}

Dr. Bhuvan Modi received PhD. degree from Prairie View A \& M University, Texas A \& M University System, in 2012. He earned his M.S. degree in Electrical Engineering from Lamar University, United States of America, M.S. degree in Electronics and Communication Engineering from Dharmsinh Desai University, India, and the B.S. degree in Electronics and Communication Engineering from North Gujarat University, India, in 2009, 2002 and 2001, respectively. He is currently working as a Senior

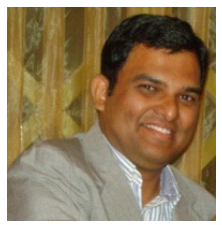
Member of Technical Staff at AT\&T Mobility Lab, Seattle, WA. He received 'Student Travel Grant Award' to present his work at the IEEE MILCOM'11. Over last few years, Mr. Modi has published over a dozen peer reviewed conference and journal articles. His current research interests include cross-layer design/optimization for adaptive-link cooperative relay networks, Software Define Networking and 4G VoLTE.

Dr. Oluwatobi O. Olabiyi received the B.Sc. degree in Electronic and Electrical Engineering from Obafemi Awolowo University, Ile-Ife and M.S. and $\mathrm{PhD}$ degree in Electrical Engineering from Prairie View A\&M University, Texas. Over the last few years, he has co-authored approximately two-dozen peer-reviewed conference and journal articles. He was the recipient of the Roy G. Perry College of Engineering Outstanding Masters Student of the Year Award (2011) and the National Society of

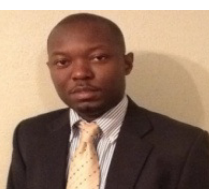
Black Engineer's Golden Torch Award for Graduate Student of Year (2012). His research interests include dynamic spectrum access, MIMO, cooperative communications, statistical signal processing, compressive sensing, machine-learning and optimization techniques.

Dr. Annamalai is presently the Director of Center of Excellence for Communication Systems Technology Research, a Texas A\&M Board of Regents approved University Research Center at the Prairie View A\&M University, and a tenured faculty member in the Department of Electrical and Computer Engineering. He has over 20 years of research/teaching experience in wireless communications at Motorola, University of Victoria, Air Force Research Laboratory, Virginia Tech and PVAMU with approximately 200 peer-reviewed publications and 5 book chapters. Dr. Annamalai

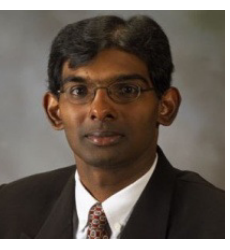
has been honored by his colleagues on numerous occasions for his excellence in research including winning the 2011 Roy G. Perry College of Engineering Outstanding Faculty (Research) Award, IEEE Leon Kirchmayer Prize Paper award, ASEE/AFOSR Summer Faculty Fellowships, NSERC Doctoral Prize, CAGS/UMI Distinguished Doctoral Dissertation Award, IEEE VTS/Motorola Daniel E. Noble Fellowship, among others. He had served on the Editorial Boards of four IEEE journals/transactions in the last 15 years, and has helped to organize a few major IEEE conferences on wireless communications including serving in the capacity of Technical Program Chair of the 2002 IEEE Vehicular Technology Conference in Vancouver, Canada. His current research interests include cooperative spectrum sensing, compressive sensing, cross-layer design for scalable multimedia transmission and cooperative wireless communications. 